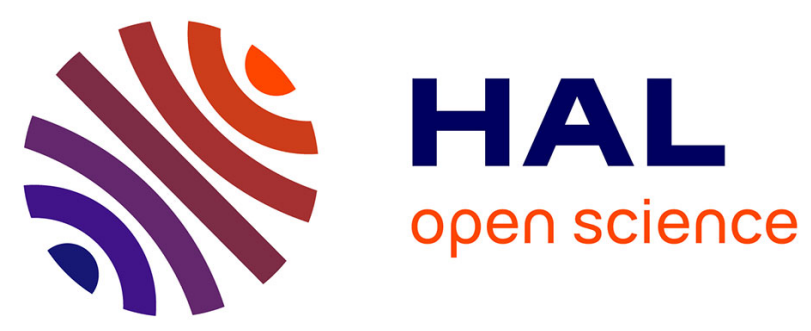

\title{
A new technique based on convolutional neural networks to measure the energy of protons and electrons with a single Timepix detector
}

Marine Ruffenach, Sébastien Bourdarie, Benedikt Bergmann, Stefan Gohl, Julien Mekki, Jean-Roch Vaillé

\section{To cite this version:}

Marine Ruffenach, Sébastien Bourdarie, Benedikt Bergmann, Stefan Gohl, Julien Mekki, et al.. A new technique based on convolutional neural networks to measure the energy of protons and electrons with a single Timepix detector. IEEE Transactions on Nuclear Science, 2021, 68 (8), pp.1746-1753. 10.1109/TNS.2021.3071583 . hal-03238974

\section{HAL Id: hal-03238974 \\ https://hal.science/hal-03238974}

Submitted on 19 Jul 2021

HAL is a multi-disciplinary open access archive for the deposit and dissemination of scientific research documents, whether they are published or not. The documents may come from teaching and research institutions in France or abroad, or from public or private research centers.
L'archive ouverte pluridisciplinaire HAL, est destinée au dépôt et à la diffusion de documents scientifiques de niveau recherche, publiés ou non, émanant des établissements d'enseignement et de recherche français ou étrangers, des laboratoires publics ou privés. 


\title{
A new technique based on convolutional neural networks to measure the energy of protons and electrons with a single Timepix detector
}

\author{
M. Ruffenach, S. Bourdarie, B. Bergmann, S. Gohl, J. Mekki, and J. Vaillé
}

\begin{abstract}
The Timepix chip has been exposed to the outer space for the first time with the SATRAM (Space Application of Timepix-based Radiation Monitor) instrument on Proba-V (Project for On-Board Autonomy Vegetation), a European Space Agency's (ESA) satellite. This study's objective is to develop a new technique to improve the separation of protons and electrons, which are detected by the single layer Timepix detector in SATRAM. The current identification method, proposed by S. Gohl et al. [1], is based on pattern recognition and stopping power measurements. In this article, the limitations of this method are discussed. A new method based on neural network trained with Geant4 data is proposed. Its validation with SATRAM data is presented. Similarly, a neural network trained with Geant4 data is introduced. Its purpose is to deduce the particles' incident energy using the energy deposited in the Timepix.
\end{abstract}

Index Terms-Geant4, Monte-Carlo simulations, neural networks, radiation belts, radiation monitor, space environment, Timepix.

\section{INTRODUCTION}

$\mathrm{P}$ ROTONS and electrons are trapped by the magnetic field of the Earth and constitute radiation belts [2]. These charged particles can interact with satellites' components and cause damages [3]. Measurements are required to develop and improve models, such as AP-8, AE-8 [4] [5], AP-9, AE-9 [6], or GREEN [7], describing the radiation environment. Indeed, this knowledge allows to predict the effects of particles on the satellites' components, and therefore to prevent radiation effect damages on electronics. In order to detect and separate electrons and protons in a broad range of energies, devices with several sensing and shielding elements are typically employed [8][12]. These radiation monitors have a mass of several kilograms which can cause difficulties to find flight

This work was conducted as part of a co-financing of doctoral thesis research CNES $\mathrm{N}^{\circ} 17054$ - ONERA $\mathrm{N}^{\circ} 9218$.

M. Ruffenach, and S. Bourdarie are with The French Aerospace Lab/Département Physique Instrumentation Environnement et Espace, ONERA, 31055 Toulouse, France (e-mail: marine.ruffenach@cnes.fr).

B.Bergmann, and S. Gohl are with Institute of Experimental and Applied Physics, Czech Technical University in Prague, Horska 3a/22, 12800 Prague 2, Czech Republic.

J. Mekki is with CNES, 31401 Toulouse, France.

J. Vaillé is with IES - UMR UM/CNRS 5214, Université de Montpellier, 34097 Montpellier, France. opportunities. This study's aim is to propose a solution to measure simultaneously, with a single sensor, a wide energy range for protons and electrons. The detector shall be compatible with small satellites such as Cubesat, thus imposing a low power consumption, and small mass and volume requirements on the instrument. The Timepix chip [13], a $256 \times 256$ pixelated silicon sensor with a thickness of $300 \mu \mathrm{m}$, is compatible with Cubesat. Several Timepix detectors flew [1] [14]-[16] e.g. on the LUCID experiment [17]. Furthermore, methods have been proposed to identify type of particles in radiation belts based on SATRAM measurements [1]. However, optimizations are required to improve these methods since some limitations have been noticed.

In section II a brief presentation of the SATRAM instrument is given, along with a short description of the previous identification methods used. In section III a new particle identification method based on neural networks is proposed. In this section, the data base creation with Geant4, as well as the neural networks training and its validation with SATRAM data are discussed. A neural network for energy recognition for protons and electrons is proposed in section IV. Finally, conclusions are given in section V.

\section{PREVIOUS PARTICLE IDENTIFICATION METHOD BASED ON SATRAM DATA AND ITS LIMITATIONS}

SATRAM [1] [14], a technology demonstrator, is the first instrument based on the use of the Timepix chip exposed to the outer space. It flew on the Proba-V satellite launched on May $7^{\text {th }}, 2013$ into a sun-synchronous orbit at an altitude of $820 \mathrm{~km}$. The SATRAM instrument consists of a Timepix chip and electronics, housed in an aluminum box. It weights a total of $380 \mathrm{~g}$ with a power consumption of $2.5 \mathrm{~W}$. The Timepix chip [13], developed by the Medipix collaboration at CERN, is a hybrid semiconductor pixel detector, made up of a $300 \mu \mathrm{m}$ thick silicon layer. Its $256 \times 256$ pixels of pitch size $55 \mu \mathrm{m}$ are operated in the Time-over-Threshold (ToT) mode. The energy deposited by ionizing particles in each pixel is registered in the square matrix of pixels read out in frames of preset acquisition time. Ionizing particles passing through the Timepix sensor leave tracks in the pixel matrix. These tracks are sets of energy depositions in neighboring pixels and are referred to as clusters. Due to the frame based architecture, incident particle tracks may overlap in the sensor, thus 
creating a single cluster. A method has been developed by S. Gohl et al. [1] to identify type of particles using the cluster shape, stopping power, cluster height, angle definition, mass and geometrical centers of the cluster. All these parameters are detailed in [1] and [18]. This identification method has been applied to SATRAM data to separate protons and electrons. World maps of proton and electron count rates obtained for SATRAM data from 10/01/2014 to $10 / 10 / 2014$ are illustrated in Fig. 1.
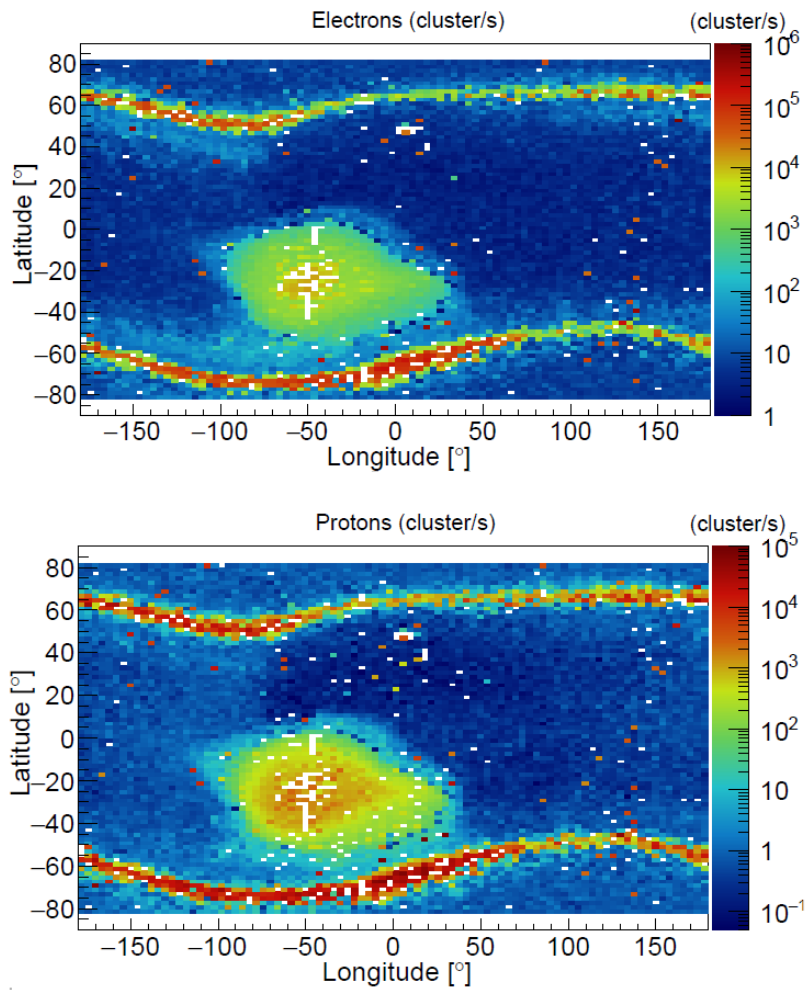

Fig. 1. Maps of count rates of electrons and protons obtained with the identification method, as described in [1], for SATRAM data from 10/01/2014 to $10 / 10 / 2014$.

The radiation belts' axis of symmetry corresponds to the Earth's magnetic dipole axis, since the particles that are in the radiation belts are trapped by the Earth's magnetic field. At the level of the South Atlantic Anomaly (SAA), protons and electrons of the radiation belts are observed at a lower altitude, as the magnetic field is inclined and eccentric. Moreover, the outer electron belt is close to the Earth near to the polar cones. These characteristics are illustrated in Fig. 2.
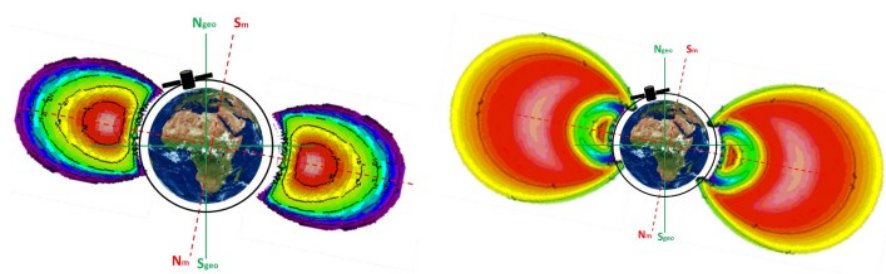

Fig. 2. A Low Earth Orbit satellite in the proton radiation belt (left) and in the electron radiation belts (right).

On satellites with a low orbit, this leads to the observation of strong electron fluxes near the poles, and of strong proton and electron fluxes at the SAA. No protons with an energy higher than several $\mathrm{MeV}$ are expected near the poles. Indeed, the Earth's magnetic field for these magnetic field lines is too weak. Due to the SATRAM instrument's shielding, protons with an energy lower than $20 \mathrm{MeV}$ cannot reach the Timepix [1]. Hence, the SATRAM instrument cannot measure protons near the poles. Therefore, regarding Fig. 1, the major part of particles seems to be correctly classified. However, protons with an energy greater than $20 \mathrm{MeV}$ are detected in large quantities near the poles while, at these locations, they cannot be trapped in the radiation belts. Such a feature does not support the radiation belt theory as measurements indicate high $>20 \mathrm{MeV}$ proton fluxes beyond the magnetic shielding location. Besides, some characteristic structures of electrons as the one at the South-West of the SAA appear in the proton fluxes world map. This highlights the necessity to come up with algorithms with a better particle separation capability. In this paper, convolutional neural networks are used to improve the particle identification.

\section{PARTICLE IDENTIFICATION METHOD BASED ON NEURAL NETWORKS TRAINED WITH GEANT4 DATA}

Convolutional neural networks (CNNs) [19] are deep learning neural networks containing at least one layer where a convolution operation is made. They are widely used to analyze arrays of data such as images. Indeed, these algorithms detect patterns in data which allow their categorization into different classes.

In most cases, tracks of protons and electrons in the Timepix are, depending on the energy and the incoming angle, quite different. CNNs detect features that are specific to an object. In particular, they may detect features characterizing tracks of protons and electrons in the Timepix, thus allowing to make the difference between both types of particles. For this purpose, CNNs need to be trained with labelled data. For particle type recognition, thousands of labelled data of protons and electrons are required.

Furnell et al. [17] have tested a CNN to classify particles registered in Timepix on-board the LUCID experiment. To train the CNN, they use a web application called LUCID Trainer where volunteers classify tracks of particles. Constituted of 1800 particle tracks and valid only for the geometry of the LUCID instrument, the training data base is thus based entirely on human classification.

It is quite difficult to label manually thousands of data. Moreover, labelling errors lead to poor learning of the neural network. A reliable data base generated automatically is required. In this work, Geant 4 is used for the training and validation datasets.

\section{A. Creation of the data base with Geant4}

The Geant4 (Geometry ANd Tracking) [20] [21] toolkit allows to perform Monte-Carlo simulations reproducing the interaction between particles and matter. The SATRAM instrument model contains the Timepix sensor, PCB's, and the aluminum box. The satellite is simplified to a block of aluminum with the corresponding stopping power. The 
complete description of the SATRAM instrument can be found in [1]. An omnidirectional radiation field was created using a spherical source surrounding the SATRAM instrument with a cosine-law angular distribution of the particles' initial velocities [22]. Ten million particles are simulated per incident energy, with 154 incident energies from $0.4 \mathrm{MeV}$ to $5 \mathrm{MeV}$ for electrons (with a step of $30 \mathrm{keV}$ ) and 161 incident energies from $17 \mathrm{MeV}$ to $200 \mathrm{MeV}$ for protons (with a step of $200 \mathrm{keV}$ from $17 \mathrm{MeV}$ to $30 \mathrm{MeV}$, a step of $1 \mathrm{MeV}$ from $30 \mathrm{MeV}$ to $100 \mathrm{MeV}$, and a step of $4 \mathrm{MeV}$ from $100 \mathrm{MeV}$ to $200 \mathrm{MeV}$ ). Protons and electrons, respectively with an energy higher than $200 \mathrm{MeV}$ and $5 \mathrm{MeV}$, are not simulated. Indeed, protons of $200 \mathrm{MeV}$ and higher produce similar tracks in the SATRAM instrument, as do electrons of $5 \mathrm{MeV}$ and higher. Also, electrons lower than $0.4 \mathrm{MeV}$ and protons lower than $17 \mathrm{MeV}$ are not simulated since they do not reach the Timepix due to the SATRAM instrument's shielding. For each particle, the deposited energy in each pixel is registered at the output of the Geant4 simulations. Heavy ions are not simulated, since only the species predominantly present in the Earth's space environment are taken into consideration. The error introduced will not be significant, as fluxes of heavy ions are very low compared to those of protons and electrons. However, this will lead to a misclassification if a track of a heavy ion is registered by the SATRAM instrument. It will be falsely classified as a proton or an electron.

The advantage of using Geant 4 to create the data base of labelled data is that the particles' type and energy are known. The data base is separated into two sets for the training phase and the validation phase. Each set contains around 4000 tracks of protons and 4000 tracks of electrons. All energies simulated by Geant 4 are used to create the training and the validation datasets, with the same number of particles taken into consideration for each incident energy. Energies chosen for simulations allow to take into account all the behaviors that a particle can have according to its direction of arrival, its type, and its energy. Each track has its corresponding label one-hot encoded (all input and output variables in machine learning algorithms must be numeric). Consequently, the corresponding label of an electron is 0 , while it is 1 for a proton (it is an arbitrary choice). All the values of deposited energies are normalized to the maximum deposited energy value in a pixel found in the validation and the training data sets. This ensures better performances of the neural networks, in particular to avoid their divergence. First, computers lose accuracy when too large and too small numbers are used for mathematical operations. Then, corrections brought to weights are more homogeneous. Indeed, using normalized data, the different features vary on similar ranges of values.

\section{B. Discrimination of protons and electrons with neural networks}

The training data set is used to train the neural network to recognize the type of particles by analyzing tracks in the Timepix. The validation data set is then used to check the accuracy of the neural network to recognize the type of particles. The CNN's architecture is based on convolution, pooling, activation, and fully connected layers. These layers are explained later in the paper. The Python library Tensorflow [23] is used to code the neural network. This tool, developed by Google, allows to build and train neural networks easily. Indeed, basic operations used in machine learning are available through optimized objects. Before training, weights of features and layers are initialized randomly. Then, they are modified at each epoch to improve the CNN's performances for the particle type identification. An epoch is a complete pass (one forward pass and one backward pass) through the entire training samples.

Training and validation sets created with Geant 4 for the SATRAM instrument are used to train the CNN. Several architectures have been tested as well as several sets of hyperparameters. Hyper-parameters represent variables determining the neural network's structure and how it is trained. Number of epochs, number of samples used during each epoch (also called batch size), type and number of layers, and also parameters of layers (number and size of filters...) are hyperparameters, as well as the learning rate which determines how quickly the model is adapted at each epoch. The best training has been obtained for a neural network composed of a convolutional layer, max-pooling layer and ReLU (Rectified Linear Units) layer, repeated twice, and two fully connected layers. This architecture is illustrated on Fig. 3.

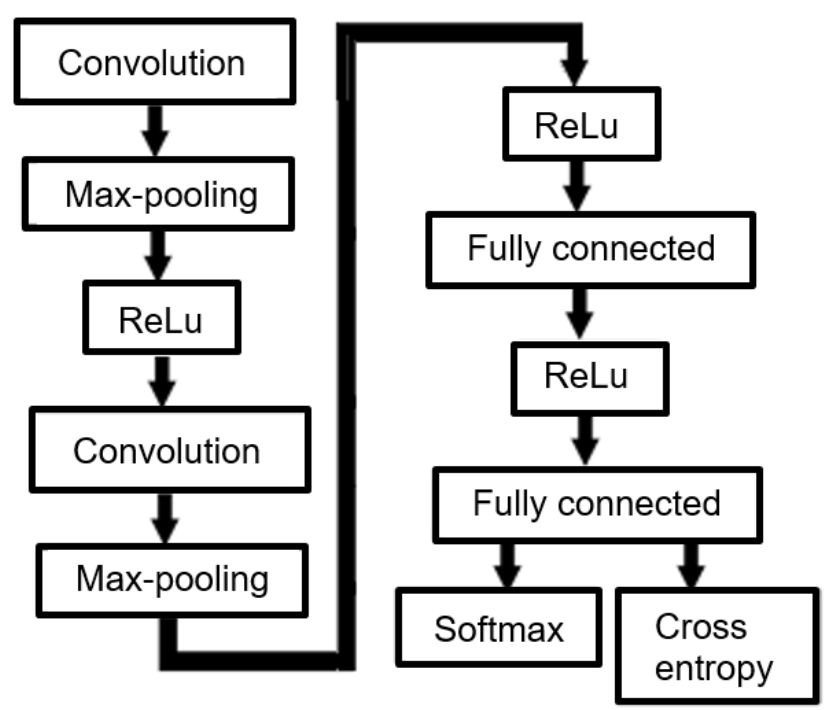

Fig. 3. Architecture of the neural network.

The convolutional layers are used to detect features. Neural networks are invariant to scale and orientation changes using the max-pooling layers [24]. Activation layers ReLU [25] introduce non-linearity which allows to learn more complex functions. This leads to better performances during the training phase. Fully connected layers [26] are used to learn complex combinations. The softmax function [27] is used in the normalization layer to calculate the probability for each class. The expression of the softmax function is given in Eq. 1 . 


$$
\operatorname{softmax}(x)_{i}=\frac{\exp \left(x_{i}\right)}{\sum_{j=1}^{C} \exp \left(x_{j}\right)}
$$

where $C$ is the number of elements of the $x$ vector, or in other words the number of classes, and $x_{i}$ the $\mathrm{i}^{\text {th }}$ component of the vector $x$. The class predicted by the CNN is the one which has the higher probability.

The cross-entropy is used to calculate the error of the neural network, that is, the difference between predicted labels and true labels. A neural network which is well trained has a crossentropy close to 0. Its expression is given in Eq. 2 .

$$
L=-\frac{1}{N} \sum_{i=1}^{N} \sum_{j=1}^{C} y_{i, j} \ln \left(p_{i, j}\right)
$$

where $C$ is the number of classes, $N$ the number of tracks that are analyzed, $y$ the label one-hot encoded, and $p$ the probabilities obtained at the softmax function output.

During each epoch, a batch of 32 tracks picked randomly in the training data set with their corresponding labels is used to train the CNN with a learning rate of $10^{-4}$. Weights are adjusted in order to have a good correspondence between the output of the CNN and the labels given for input tracks. Once weights are adjusted, the CNN is tested on the entire validation set by comparing the outputs given by the neural network and their corresponding labels. The classification accuracy of the CNN is then computed. This process is repeated each time until the neural network has learned correctly. The learning phase ends when the accuracy of the identification is stabilized. It is not necessary to continue learning when the accuracy does not evolve, otherwise it could cause over-fitting (the network has memorized the training data and fails to generalize). The accuracy as well as the error calculated using the cross-entropy are illustrated on Fig. 4.
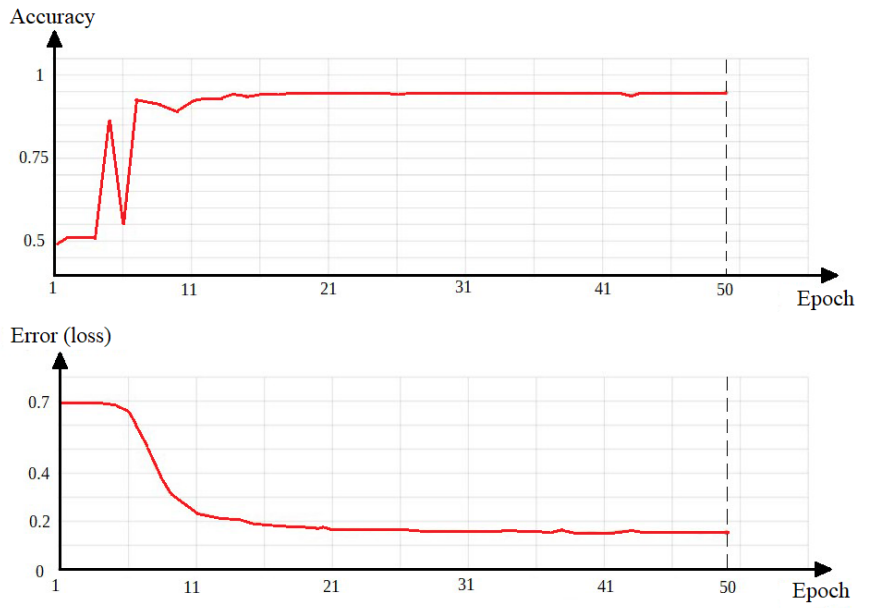

Fig. 4. Evolution of the accuracy (top) and of the error (bottom) as a function of the number of epoch for the validation set.

The error value for the validation set continuously decreases during the training phase, until it reaches a limit value. This means that the neural network learns correctly, thus excluding the case of over-fitting, the case of under-fitting (the network learns and generalizes but the optimal value has not yet been reached), as well as the case of non-learning (the network does not learn and does not generalize). After 50 epochs the accuracy stabilizes at $94.79 \%$ for the validation set, which is a good result in the case of discriminating the types of particles in radiation belts. This means that the neural network is able to correctly differentiate between protons and electrons in $94.79 \%$ of cases, regardless of their energy and angle of incidence. Results are illustrated using a normalized confusion matrix given in Fig. 5.

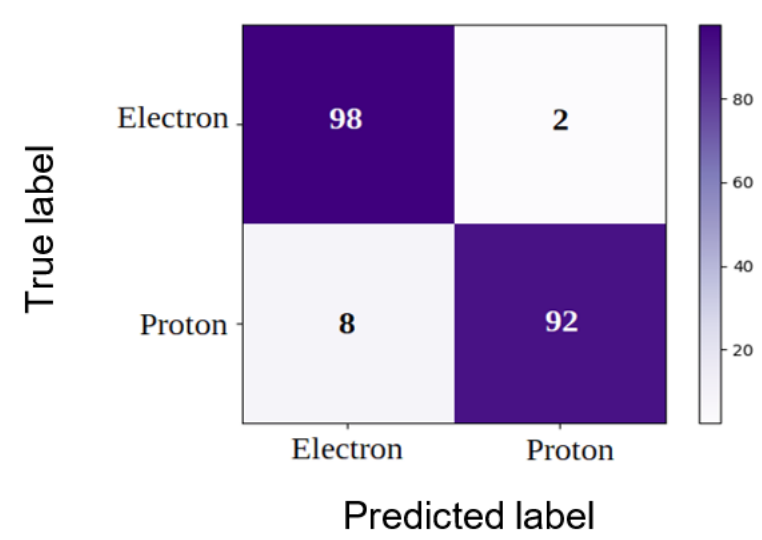

Fig. 5. Normalized confusion matrix for the type recognition. The numbers are in percent.

This means that the neural network is able to discriminate electron tracks in $98 \%$ of cases, and proton tracks in $92 \%$ of cases. The next step is to apply this neural network to real data.

\section{Validation of type recognition performed by neural networks with in-flight data}

Since the complete geometry of the SATRAM instrument as well as the satellite have been taken into consideration to perform Geant4 simulations, simulated tracks and real tracks must be similar. The aim of this section is to apply the neural network trained with Geant 4 data to in-flight measurements performed by the SATRAM instrument on-board the Proba-V satellite. SATRAM data are analyzed for ten days from $10 / 01 / 2014$ to $10 / 10 / 2014$. Consecutive frames have an exposure time of $20 \mathrm{~s}, 200 \mathrm{~ms}$, and $2 \mathrm{~ms}$. Only the frames with an occupancy lower than $20 \%$ are considered, otherwise the large number of overlaps from various tracks makes the analysis with the neural network impossible. Indeed, clusters are composed of neighbour pixels. If two tracks overlap, they will falsely be considered as a single cluster. Therefore, this situation must be reduced by analyzing frames with an occupancy lower than $20 \%$. In addition, if a particle deposits energy in at least one pixel on the edges of the Timepix matrix, it is not taken into account. Indeed, truncated tracks are not treatable and lead to a bad classification. In addition to the measurements made by the Timepix, the satellite's position (longitude, latitude, and altitude) is available for each frame. 
For each frame, each cluster is extracted individually. The deposited energy is normalized by the maximum value of deposited energy in a pixel obtained with Geant4 data. Normalized clusters are then analyzed by the neural network which produces an output corresponding to the particle's type. This allows establishing maps of proton and electron count rates, expressed in number of clusters per second, obtained by neural network analysis for the SATRAM data. Results are given in Fig. 6.
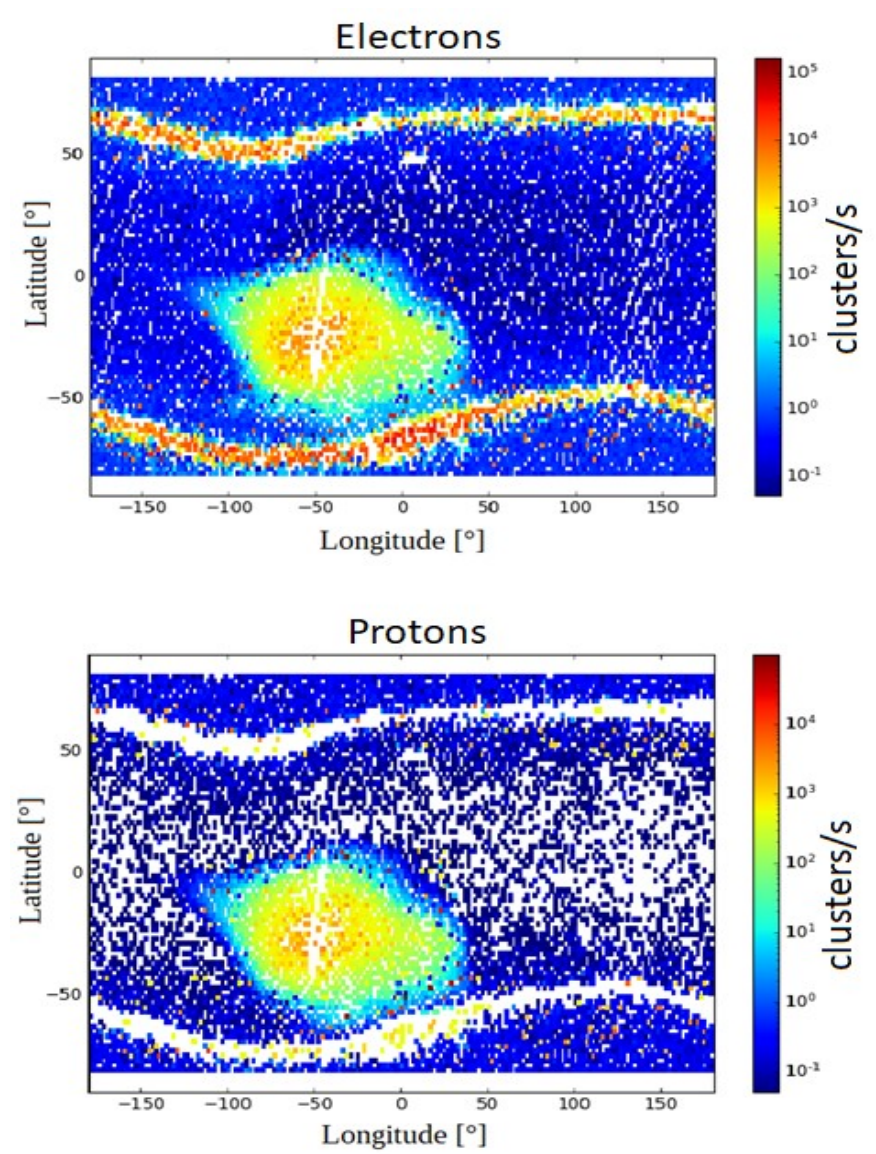

Fig. 6. Maps of electron (top) and proton (bottom) count rates obtained with the CNN applied to SATRAM data from 10/01/2014 to 10/10/2014.

In Fig. 6, electrons are mostly observed near the poles at high latitudes and in the SAA, while protons are mostly found in the SAA. Some protons can be found near the poles, which is probably due to errors during the training phase. Moreover, particles are detected outside the SAA and the poles, especially in the case of electrons. Only particles predominantly present in radiation belts are taken into account during the training phase of the neural network. The CNN is not trained to recognize protons with an energy higher than $200 \mathrm{MeV}$ nor heavy ions $(Z>1)$. When analyzing the data acquired by the SATRAM instrument, if a track is produced by these particles, it is identified by default as being a proton or an electron. Then, the ions can also interact with the shielding of the SATRAM instrument and produce secondary particles, which are classified as electrons. However, particles fluxes detected outside the SAA and the poles are very low, even negligible, compared to those observed for protons and electrons in radiation belts. That is why the simulation of ion tracks to train the neural network is not considered in this paper. Some tracks measured outside the poles and the SAA classified as electron tracks are illustrated on Fig. 7.
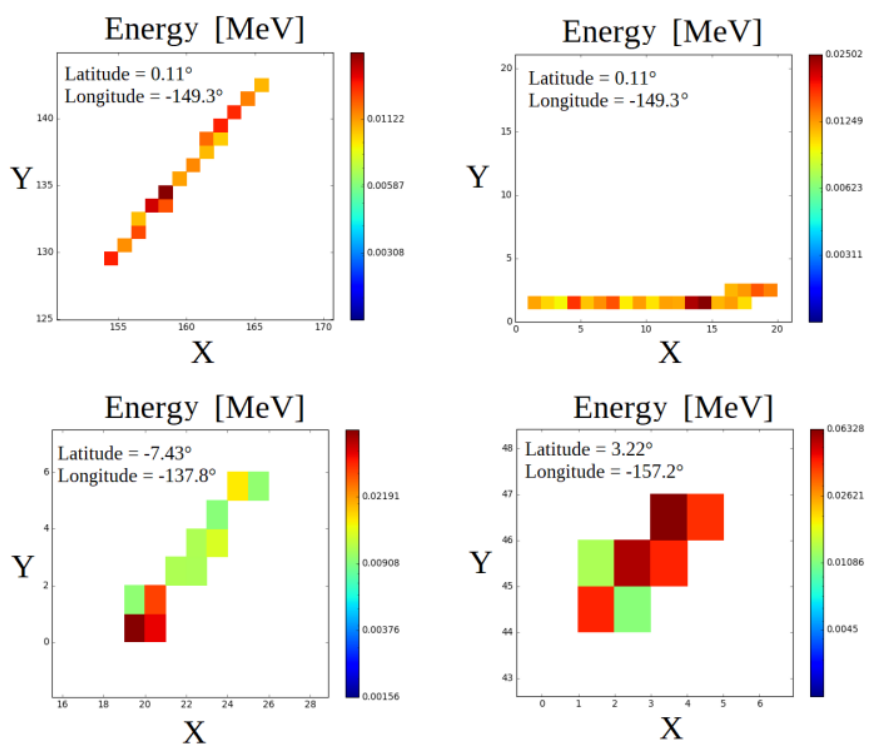

Fig. 7. Tracks detected outside the SAA and the poles classified as electrons by the neural network.

Tracks shown in Fig. 7, classified as being produced by the interaction of electrons in the Timepix, may have actually been made by ions, including energetic protons from cosmic radiations. These tracks are indeed linear, which is a characteristic usually attributed to the tracks produced by protons rather than those produced by electrons. The low fluxes measured outside the SAA and the poles for the proton and electron maps are generally attributed to the product of cosmic rays, and not to protons and electrons trapped in radiation belts.

Blanks observed for proton measurements near the poles and outside the SAA in Fig. 6 are not due to a lack of data but to the fact that the neural network has classified all tracks as electrons. Blanks observed at the middle of the SAA for protons and electrons are due to the processing of frames with an occupancy lower than $20 \%$. Indeed, very high fluxes are observed inside the SAA and thus the occupancy is higher than $20 \%$ for this region, even by considering $2 \mathrm{~ms}$ exposure time frames.

Results shown in Fig. 6 are encouraging. Indeed, protons and electrons are quite well distinguished by the neural network. This can be supported by other measurements. Maps of electron measurements made by the ICARE-NG [8] [9] (Influence sur les Composants Avancés des Radiations de l'Espace-Nouvelle Génération) instrument on-board the SACD (Satellite for Scientific Applications-D) satellite are shown on Fig. 8, and maps of proton measurements made by the EPT [28] (Energetic Particle Telescope) instrument on-board Proba-V satellite are illustrated on Fig. 9. 


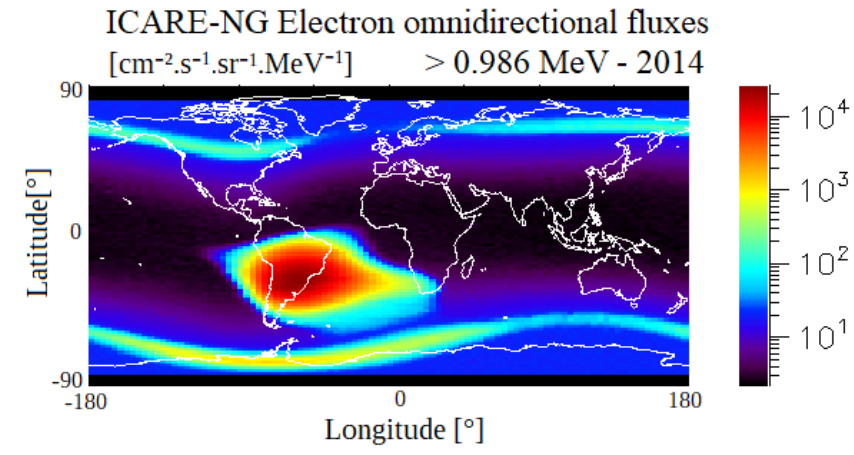

Fig. 8. ICARE-NG electron fluxes for energies higher than $0.986 \mathrm{MeV}$ in 2014.

EPT Proton unidirectional fluxes $\left[\mathrm{cm}^{-2} \cdot \mathrm{s}^{-1} \cdot \mathrm{sr}^{-1} \cdot \mathrm{MeV}^{-1}\right]$

$13 \mathrm{MeV}-29 \mathrm{MeV}$ from 01/01/2015 to 06/01/2015

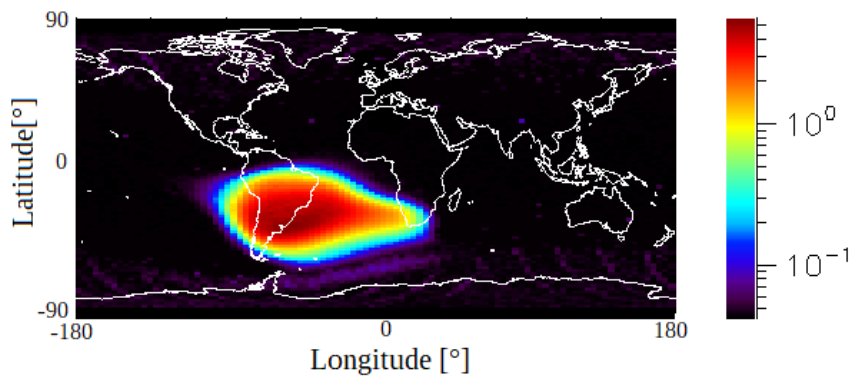

Fig. 9. EPT proton fluxes for energies between $13 \mathrm{MeV}$ and $29 \mathrm{MeV}$ from January 2015 to June 2015.

In Fig. 9, protons are only measured in the SAA while electrons are measured both in the SAA and near the poles in Fig. 8. These types of maps are produced by the analysis of SATRAM data with the neural network.

\section{ENERGY RECOGNITION BASED ON NEURAL NETWORKS FOR PROTONS AND ELECTRONS}

The CNN developed in the previous section allows identifying particles' type, i.e. protons and electrons. To go further, CNNs are tested to deduce from the tracks, in addition to the type, the incident energy of particles. The incident energy corresponds to the energy of the particle before it interacts with the instrument.

A CNN is developed with an architecture identical to the one used for the particle identification, but with different hyper-parameters, to perform energy identification for protons. Data are divided into 10 classes in the energy range from 17 to $200 \mathrm{MeV}$.

The neural network is trained with Geant 4 data. The same Geant 4 output files are used to create the training and the validation data sets. For each class, around 400 proton tracks are considered for the training data set and likewise, 400 proton tracks for the validation data set. All energies simulated by Geant 4 are used to create the training and the validation data sets, with the same number of particles taken into consideration for each class. Results obtained after the training of the CNN are illustrated in Fig. 10 using normalized confusion matrix.

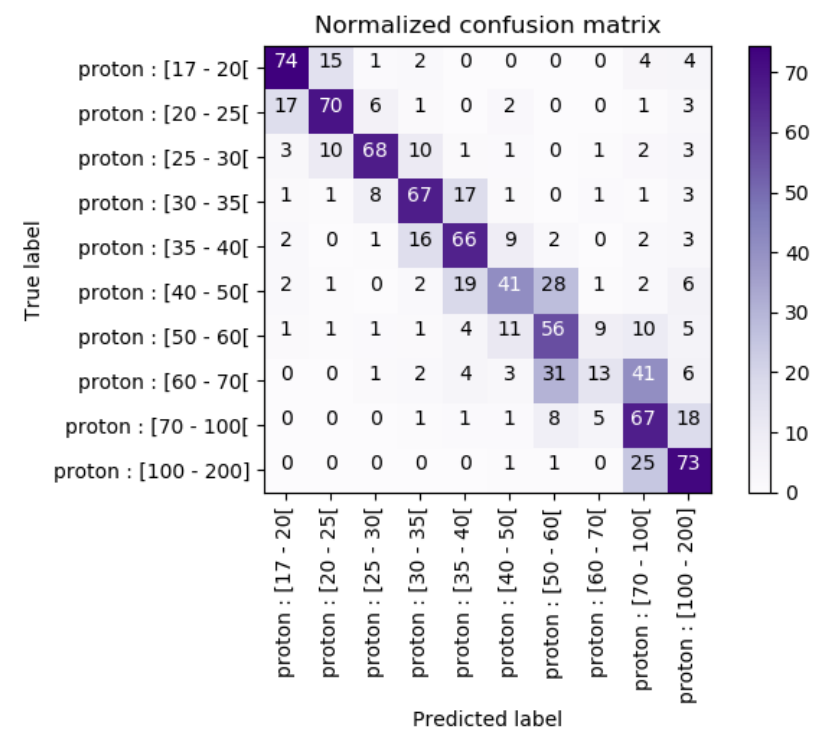

Fig. 10. Confusion matrix for the energy recognition in the case of protons.

In Fig. 10 a good distinction between energy classes can be noticed. However, most of errors come from neighbour classes. For instance, a $50 \mathrm{MeV}$ proton produces in the Timepix a similar track as the one produced by a $49 \mathrm{MeV}$ proton. That is why errors between neighbour classes are observed.

This trained neural network is used on SATRAM data to calculate proton fluxes. Tracks detected as protons by the neural network of type recognition are injected in this neural network for energy recognition. Fluxes of protons for an energy higher than $69 \mathrm{MeV}$ (integrated fluxes above $69 \mathrm{MeV}$ ) are calculated. This energy range is chosen to be compared with ICARE-NG measurements on-board the JASON-2 satellite at an altitude of $1336 \mathrm{~km}$ [9]. Proba-V and JASON-2 are not on the same orbit. However, a tool developed by ONERA has been used to project fluxes measured by ICARENG on-board JASON-2 as if they were performed on the same orbit as Proba-V [29]. Results are illustrated on Fig. 11 for ICARE-NG measurements.

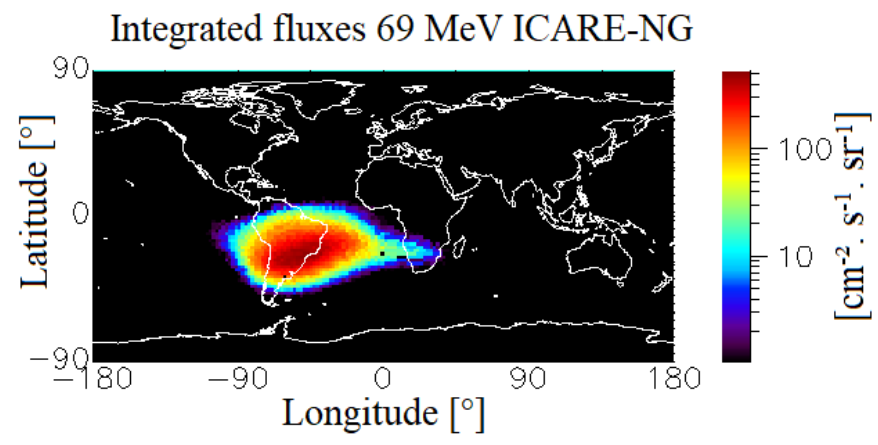

Fig. 11. Fluxes of protons with an energy higher than $69 \mathrm{MeV}$ measured by ICARE-NG on-board the JASON-2 satellite projected at an altitude of $820 \mathrm{~km}$.

Fluxes obtained by neural networks applied to SATRAM measurements are illustrated on Fig. 12. Fluxes are expressed in same units as those of ICARE-NG by dividing by the surface of the Timepix, by the exposure time, and by the solid 
angle $4 \pi$.

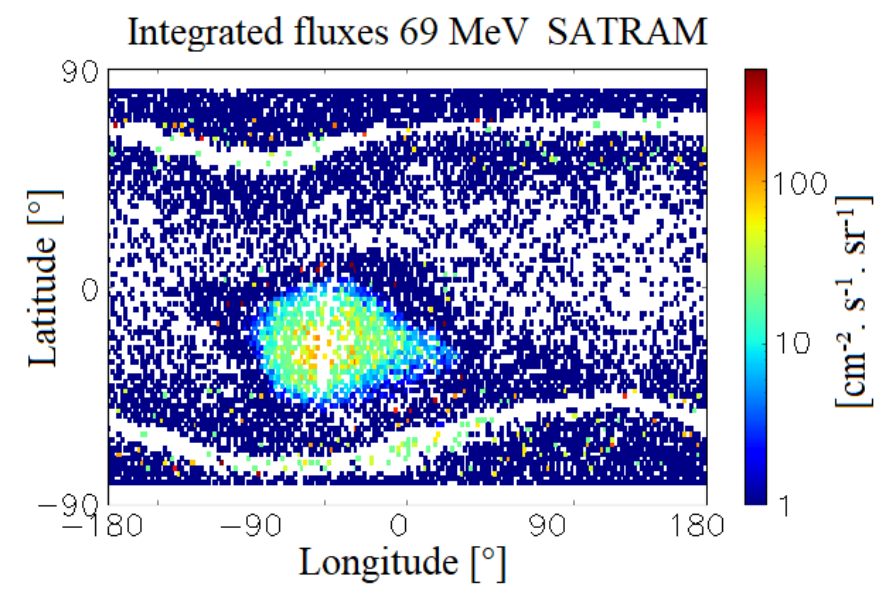

Fig. 12. Fluxes of protons with an energy higher than $69 \mathrm{MeV}$ measured by SATRAM on-board the Proba-V satellite using the neural network.

Since proton fluxes are mostly observed in the SAA, they are calculated for a latitude of $-30^{\circ}$ for both instruments for a better comparison. Proton fluxes at a latitude of $-30^{\circ}$ are illustrated on Fig. 13 for SATRAM and ICARE-NG measurements.

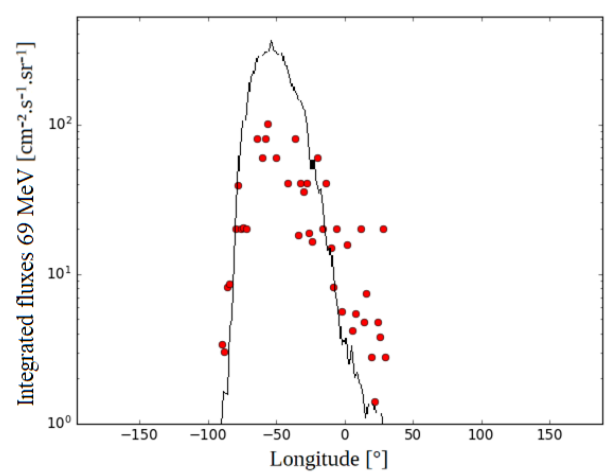

SATRAM • ICARE-NG -

Fig. 13. Proton fluxes for energies higher than $69 \mathrm{MeV}$ measured by ICARENG and SATRAM at a latitude of $-30^{\circ}$.

Globally, fluxes calculated for the SATRAM instrument and those obtained by ICARE-NG measurements projected on the Proba-V orbit are similar, except at the middle of the SAA, for a longitude close to $-50^{\circ}$. In particular, fluxes of protons with an energy higher than $69 \mathrm{MeV}$ measured by SATRAM are around $100 \mathrm{~cm}^{-2} \cdot \mathrm{s}^{-1} \cdot \mathrm{sr}^{-1}$ for a latitude of $-30^{\circ}$ and a longitude of $-50^{\circ}$, while those measured by the ICARE-NG instrument are around $400 \mathrm{~cm}^{-2} \cdot \mathrm{s}^{-1} \cdot \mathrm{sr}^{-1}$. In these regions, fluxes are very high resulting in a low quantity of tracks that can be analyzed due to the limitation of frames with an occupancy lower than $20 \%$. Due to this limitation, statistical errors occur for these regions, which explains why fluxes measured by the SATRAM instrument are lower than those measured by ICARE-NG at the middle of the SAA.

Energy recognition has also been tested for electrons. However, whatever the architecture of the neural network and hyper-parameters, the accuracy is very low. Different classes have been tested to train the neural network. However, results are not conclusive, probably due to the high diffusion of electrons in matter and all arrival directions which lead to similar tracks even if the incident energy is different. Other techniques have to be applied in order to be able to recover electrons' energy. For example, it is possible to apply the same technique than the one used for most of the radiation monitors such as ICARE-NG. This method is based on the calculation of proton and electron response functions using Geant 4 and the use of counts measured by an instrument [8] [9] [30] [31]. This will allow determining fluxes of electrons with an energy higher than a certain value, that is integrated fluxes.

\section{CONCLUSION}

A new technique based on convolutional neural network has been proposed to identify types of particles using the tracks registered with Timepix. A first neural network allows to discriminate, in $95 \%$ of cases, proton tracks from electron tracks. Its application on SATRAM data on-board the Proba-V satellite leads to better results than those obtained with other techniques. Maps of electron and proton fluxes produced by the CNN are similar to those obtained with the bigger instruments commonly used. A second CNN used for the energy recognition produces encouraging results for protons. A good training can be noticed, even if errors are observed for neighbour classes. Its application on SATRAM data to calculate fluxes of protons for energies higher than $69 \mathrm{MeV}$ leads to similar measurements than those performed with the ICARE-NG instrument, except at the middle of the SAA where high fluxes are observed, which leads to statistical errors. However, fluxes calculated for SATRAM and ICARENG are of the same order of magnitude. Energy recognition for electrons seems to be more complicated due to their high diffusion in matter. However, solutions can be brought with the calculation of response functions to calculate integrated fluxes, the use of dE-E detectors, or stopping filters.

A new version of the Timepix is now available, Timepix 3 [32]. This new version allows to measure simultaneously the energy deposited by particles in pixels and their arrival time. The use of the Timepix 3 to measure protons and electrons in radiation belts could lead to better measurements, since frames with an occupancy higher than $20 \%$ could be analyzed using the arrival time of the particles.

Many other types of neural networks exist and can be tested to measure protons and electrons with a single Timepix detector. In their paper, Furnell et al. [17] tested for example the classification of particles using a small number of features (number of pixels, curvature radius, density, width...) calculated for each track. It would be interesting in a further work to test this type of classification, as well as other classification techniques, with the use of Geant 4 for the training phase and the validation phase.

Several radiation monitors based on the use of the Timepix detector are developed such as MIRAM (Miniaturized Radiation Monitor) [33] which will be launched on the GOMX-5 mission in 2022. Thus, much more data will be available for the comparison of different data analysis algorithms and radiation models. 


\section{ACKNOWLEDGEMENT}

The SATRAM data were provided by the Institute of Experimental and Applied Physics, Czech Technical University in Prague in collaboration with ONERA.

\section{REFERENCES}

[1] S. Gohl et al., "Study of the radiation fields in LEO with the Space Application of Timepix Radiation Monitor (SATRAM)," Adv. Space Res., vol. 63, no. 5, pp. 1646-1660, Mar. 2019.

[2] S. Bourdarie and D. Boscher, "Space radiation environment," in Space Radiation Environment and its Effects on Spacecraft Components and Systems SREC04, Toulouse: Cépaduès, 2004, pp. 57-82.

[3] D. Baker, "The occurrence of operational anomalies in spacecraft and their relationship to space weather," IEEE Trans. Plasma Sci., vol. 28 , no. 6, pp. 2007-2016, Dec. 2000.

[4] D. M. Sawyer and J. I. Vette, "AP-8 Trapped Proton Environment for Solar Maximum and Solar Minimum," NSSDC WDC-A-R\&S 76-06, Dec. 1976.

[5] J. I. Vette, "The NASA/National Space Science Data Center Trapped Radiation Environment Model Program (1964- 1991)," NSSDC/WDCA-R\&S 91-29, Nov. 1991.

[6] G. P. Ginet et al., "AE9, AP9 and SPM: New Models for Specifying the Trapped Energetic Particle and Space Plasma Environment," Space Sci. Rev., vol. 179, no. 1-4, pp. 579-615, Nov. 2013.

[7] A. Sicard, D. Boscher, S. Bourdarie, D. Lazaro, D. Standarovski, and R. Ecoffet, "GREEN: the new Global Radiation Earth ENvironment model (beta version)," Ann. Geophys., vol. 36, no. 4, pp. 953-967, Jul. 2018.

[8] D. Boscher et al., "In-Flight Measurements of Radiation Environment on Board the Argentinean Satellite SAC-D," IEEE Trans. Nucl. Sci., vol. 61, no. 6, pp. 3395-3400, Dec. 2014.

[9] D. Boscher et al., "In Flight Measurements of Radiation Environment on Board the French Satellite JASON-2," IEEE Trans. Nucl. Sci., vol. 58, no. 3, pp. 916-922, Jun. 2011.

[10] K. Yando, R. M. Millan, J. C. Green, and D. S. Evans, “A Monte Carlo simulation of the NOAA POES medium energy proton and electron detector instrument: Technique," J. Geophys. Res., vol. 116, no. A10231, Oct. 2011.

[11] D. G. Mitchell et al., "Radiation belt storm probes ion composition experiment (RBSPICE)," in The Van Allen Probes Mission, N. Fox and J. L. Burch, Eds. Boston, MA, USA: Springer, 2014, pp. 263-308.

[12] J. B. Blake et al., "The magnetic electron ion spectrometer (MagEIS) instruments aboard the radiation belt storm probes (RBSP) spacecraft," in The Van Allen Probes Mission, N. Fox and J. L. Burch, Eds. Boston, MA, USA: Springer, 2014, pp. 383-421.

[13] X. Llopart et al., "Timepix, a 65k programmable pixel readout chip for arrival time, energy and/or photon counting measurements," Nucl. Instrum. Methods Phys. Res. Sect. Accel. Spectrometers Detect. Assoc. Equip., vol. 581, no. 1, pp. 485-494, Oct. 2007.

[14] C. Granja et al., "The SATRAM Timepix spacecraft payload in open space on board the Proba-V satellite for wide range radiation monitoring in LEO orbit," Planet. Space Sci., vol. 125, pp. 114-129, Jun. 2016.

[15] T. Baca et al., "Miniaturized X-ray telescope for VZLUSAT-1 nanosatellite with Timepix detector," $J$. Inst., vol. 11, no. 10, pp. C10007, Oct. 2016.

[16] D. Turecek et al., "Small dosimeter based on timepix device for international space station", J. Inst., vol. 6, pp. C12037, Dec. 2011.

[17] W. Furnell et al., "First results from the LUCID-Timepix spacecraft payload onboard the TechDemoSat-1 satellite in Low Earth Orbit," Adv. Space Res., vol. 63, no. 5, pp. 1523-1540, Mar. 2019.

[18] S. Gohl et al., "Measurement of particle directions in low earth orbit with a Timepix," J. Inst., vol. 11, no. 11, p. C11023, Nov. 2016.

[19] Y. Lecun and Y. Bengio, "Convolutional networks for images, speech, and time series," The handbook of brain theory and neural networks, vol. 3361, no. 10, pp. 255-258, 1995.

[20] S. Agostinelli et al., "Geant4-a simulation toolkit," Nucl. Instrum.
Methods Phys. Res. A, Accel. Spectrom. Detect. Assoc. Equip., vol. 506, no. 3, pp. 250-303, Jul. 2003.

[21] J. Allison et al., "Geant4 developments and applications," IEEE Trans. Nucl. Sci., vol. 53, no. 1, pp. 270-278, Feb. 2006.

[22] Normalisation Moddeling Sources. Accessed: Oct. 2, 2020. [Online]. Available: http://geant4.in2p3.fr/2007/prog/GiovanniSantin/GSantin Ge ant4_Paris07_Normalisation_v007.pdf.

[23] M. Abadi et al., "TensorFlow: Large-Scale Machine Learning on Heterogeneous Distributed Systems," ArXiv160304467 Cs, Mar. 2016.

[24] N. Akhtar and U. Ragavendran, "Interpretation of intelligence in CNNpooling processes: a methodological survey," Neural Comput \& Applic, Jul. 2019.

[25] A Gentle Introduction to the Rectified Linear Unit (ReLU). Accessed: Dec. 5, 2019. [Online]. Available: https://machinelearningmastery.com/ rectified-linearactivation-function for-deep-learning-neural-networks/.

[26] Fully Connected Layers in Convolutional Neural Networks: The Complete Guide. Accessed: Jun. 10, 2020. [Online]. Available: https://missinglink.ai/guides/convolutional-neural-networks/fullyconnec ted-layers-convolutional-neural-networks-complete-guide/.

[27] W. Liu, Y. Wen, Z. Yu, and M. Yang, "Large-Margin Softmax Loss for Convolutional Neural Networks," in Proc. 33rd Int. Conf. Int. Conf. Mach. Learn., vol. 48, pp. 507-516, Dec. 2016.

[28] M. Cyamukungu et al., "The Energetic Particle Telescope (EPT) on Board PROBA-V: Description of a New Science-Class Instrument for Particle Detection in Space," IEEE Trans. Nucl. Sci., vol. 61, no. 6, pp. 3667-3681, Dec. 2014.

[29] C. Inguimbert et al., "In-Flight Dark Current Nonuniformity Used for Space Environment Model Benchmarking," IEEE Trans. Nucl. Sci., vol. 65, no. 8, pp. 1676-1684, Aug. 2018.

[30] M. Ruffenach et al., "Proton Radiation Belt Anisotropy as Seen by ICARE-NG Head-A," IEEE Trans. Nucl. Sci., vol. 66, no. 7, pp. 17531760, Jul. 2019.

[31] M. Ruffenach et al., "A proton sensor for energies from $2 \mathrm{MeV}$ to $20 \mathrm{MeV}$," IEEE Trans. Nucl. Sci., vol. 67, no. 7, pp. 1351-1359, Jul. 2020.

[32] T. Poikela et al., "Timepix3: a 65K channel hybrid pixel readout chip with simultaneous ToA/ToT and sparse readout," J. Inst., vol. 9, no. 05, pp. C05013-C05013, May 2014.

[33] S. Gohl et al., "Design Study of a New Miniaturized Radiation Monitor Based on Previous Experience with the Space Application of the Timepix Radiation Monitor (SATRAM)," IEEE Nuclear Science Symposium and Medical Imaging Conference Proceedings (NSS/MIC), pp. 1-7, Sept. 2019. 